\title{
Predicting Roundabout Lane Capacity using Artificial Neural Networks
}

\author{
Apostolos Anagnostopoulos, Fotini Kehagia*, Efterpi Damaskou, Anastasios Mouratidis and Georgios \\ Aretoulis
}

Highway Laboratory, Dept. of Civil Engineering, Aristotle University of Thessaloniki, 54124, Greece

Received 31 July 2019; Accepted 18 November 2021

\begin{abstract}
Several roundabout capacity methods and approaches have been proposed until now. They are mainly based either on regression equations of observed capacity and gap acceptance or on stochastic models through simulation techniques. However, all of them rely on different variables and factors and are being adapted to local driving behavior. Hence, it is not clear if existing techniques are appropriate for reliable capacity estimations and optimal design of Greek roundabouts. This paper presents the results of an experimental research that has been conducted as a first step in the optimization of roundabouts capacity estimation, based on a dataset of 11 roundabouts in Greece. The study firstly aims to understand what geometric roundabout features and driving behavior parameters influence capacity. Artificial neural networks (NN) were tested and developed to predict accurate roundabout capacities. Appropriate roundabouts were selected for the analysis and their operational performance was filmed using a UAV and a stabilized camera during peak periods. Video image processing techniques and algorithms allowed the extraction of empirical data (traffic flows, gap acceptance parameters) and accurate geometric characteristics. The results demonstrate that artificial neural networks can predict the capacity of roundabouts accurately.
\end{abstract}

Keywords: Roundabouts, Artificial Neural Networks, Capacity, Driving Behavior, Capacity Models, UAVs.

\section{Introduction}

Roundabouts have gained increased political acceptance worldwide during the last decades and are considered as the most appealing alternative of at-grade intersections. Their effectiveness to road safety issues [1] is the main reason of the widespread of these design solutions. A significant number of before-and-after studies of roundabout conversions, indicate that modern roundabouts are safer than previous intersection treatments and the number of the fatality rate can be reduced up to 95 percent [2-5].

Except for the effectiveness of these types of intersections to road safety performance, the benefits on other aspects are great of importance as well $[1,3]$. Well-designed roundabouts improve operational performance and offer other benefits such as aesthetics and air quality.

The design of the geometry of a roundabout involves choosing between trade-offs of safety and capacity. The appropriate selection of the geometric parameters for the roundabouts design can enhance the operational performance. It is essential for transport planners and traffic engineers to determine if an existing or a proposed roundabout is a feasible layout regarding traffic operation. The evaluation of roundabouts performance is a main subject on the scientific research [4-9].

Accurate roundabout capacity models are essential for optimal roundabout designs. There exist many state-of-the-art approaches and roundabout capacity methods in the literature [10]. However, despite the similarities on the operation of roundabouts worldwide, there can be significant differences in predicted values among the existing roundabout capacity

*E-mail address: fkehagia@civil.auth.gr

ISSN: 1791-2377 @ 2021 School of Science, IHU. All rights reserved. doi:10.25103/jestr.145.24 models. Existing models differ on explanatory factors and variables. Moreover, driving behavior on roundabouts is different among countries. Greek drivers seem to be displaying hesitation and erratic behavior in the use of roundabouts [11].

Upon this, the current context which is characterized not only by gradual changes in vehicles characteristics but also by driver's behavior, shows that the dominant capacity models may not applicable today. Hence, it is anticipated that the selection of the analysis method and of the input data can give variation in capacity estimates and affect the selected roundabout design.

Roundabout design practices are still in an early stage in Greece. The operational performance analysis is based on existing practices and guidelines from the U.S.A. As the transferability of these capacity models through calibration measures is under research [11], there is a need for a better understanding of what are the factors and variables that affect capacity and how predictions can be optimized.

The aim of this paper is to understand what geometric roundabout features and driving behavior parameters influence capacity. Upon this, prediction models using artificial neural networks (NN) were tested and developed to predict accurate roundabout capacities.

\section{Roundabout Lane Capacity}

The entry lane capacity is defined by the maximum traffic flow that can be accommodated by the lane of the roundabout. It is not desirable for a roundabout to operate at its capacity.

There exist many state-of-the-art approaches and roundabout capacity methods, including different explanatory factors and variables [10]. Hence, it is anticipated that the 
selection of the analysis method and of the input data can give variation in capacity estimates and affect the selected roundabout design.

Several roundabout capacity models have been developed worldwide following various methods and approaches. They can be classified in the following three main categories:

I. Empirical capacity models. This type of model is based on relationships among the observed capacity and the geometric characteristics of the roundabouts.

II. Analytic capacity models or Gap acceptance models. This type of model is based on driving behavior and the time gaps among vehicles.

III. Stohastic models. This type of model is based on microscopic simulation software that simulate vehicles interactions.

\section{Data Collection}

\subsection{Site Selection}

Specific criteria were followed to select the most efficient sites for the data collection process and analysis. Location characteristics and design elements of the intersections were considered. The final obtained data resulted in a representative database of various roundabouts in Greece.

The main criterion for the selection process of the examined roundabouts, was the identification of capacity conditions on at least one entry of the roundabouts during the day. The presence of queued conditions verified this criterion. The selected roundabouts vary in their geometric features and location characteristics. Single lane and multilane roundabouts of various geometric elements, located in different cities, were considered for the analysis. The final selected roundabouts are presented in Table 1.

Table 1. Examined roundabouts.

\begin{tabular}{c|c|c|c|c}
\hline S/N & Roundabout & Location & Coordinates & Type \\
\hline 1 & VOL01 & Volos & $39.362256,22.938246$ & Multi-lane \\
2 & LAR01 & Larissa & $39.641190,22.412491$ & Multi-lane \\
3 & LAR02 & Larissa & $39.650051,22.409391$ & Multi-lane \\
4 & THE01 & Thermi & $40.538784,23.013318$ & Single-lane \\
5 & KAT01 & Katerini & $40.274306,22.513432$ & Single-lane \\
6 & KAT02 & Katerini & $40.269327,22.524126$ & Multi-lane \\
7 & COR01 & Corfu & $39.625695,19.910755$ & Multi-lane \\
8 & PYL01 & Pylaia & $40.558121,22.993023$ & Single-lane \\
9 & KOZ01 & Kozani & $40.301845,21.794635$ & Single-lane \\
10 & KOZ02 & Kozani & $40.297238,21.785343$ & Multi-lane \\
\hline
\end{tabular}

\subsection{The Equipment}

Two types of equipment were used to perform the experiments described in this paper to acquire reliable traffic data and extract the geometric features of the selected roundabouts:

a) a quadcopter UAV and

b) a video camera attached to a tripod.

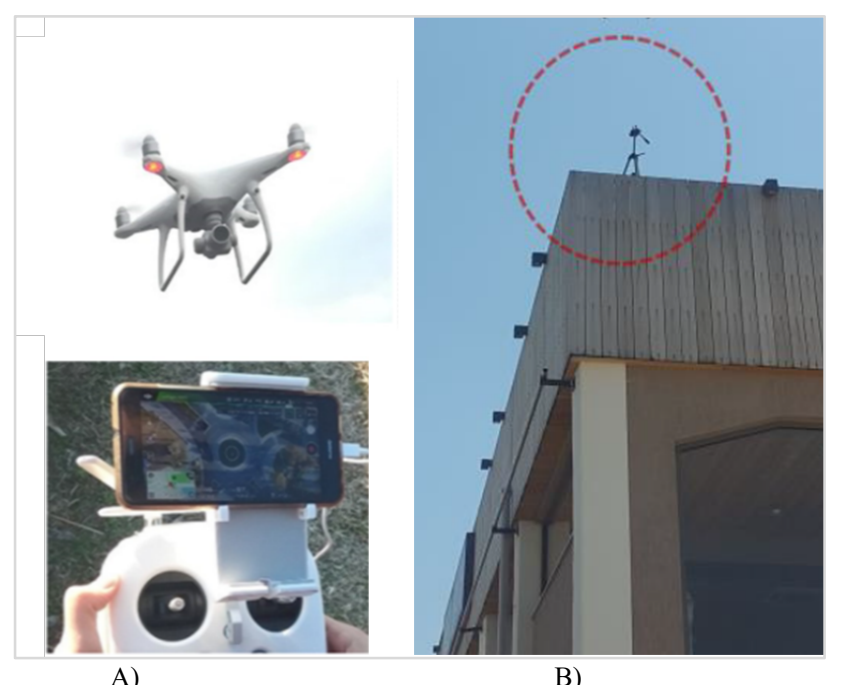

Fig. 1. The equipment.

The benefits of deploying UAVs for traffic monitoring and analysis have been considered in several studies in the past few years [12-17]. According to [17], as the camera is in the air, the drivers' attitude is not distracted by the equipment.
As a result, the extracted data represent naturalistic driving behavior, which is crucial to study the capacity of roundabout entry lanes. Moreover, UAVs provide a wide field of view, which is efficient for monitoring multiple entries of roundabouts. However, there are many factors that influence the performance of this process. Among them, weather conditions (e.g. rain), technical issues (e.g. low battery duration) and regulatory issues (e.g. no-fly zones) can significantly affect the traffic surveys. Thus, a video camera attached to a tripod was used to provide reliable traffic data as well.

\subsection{Field Data Collection}

Roundabouts' performance was recorded with the use of the equipment described above. Two types of data were collected: (a) data rely on the geometric features of the selected roundabouts and (b) traffic flow data.

Field measurements were selected to be conducted during peak periods, to ensure saturated traffic conditions. Desktopbased reconnaissance using live traffic information [18] and field observations were used to identify the peak time periods and the congested roundabout entries to be monitored.

\subsubsection{Geometric variables}

High-resolution (4096x2160) images were acquired using the UAV in specific flight altitude and vertical point of view. Low flight altitudes (roundabout layout view), high-resolution frames and GCPs of well-known coordinates increased the accuracy of the acquired images.

The geometric features of the roundabouts that that were filmed with the video camera attached to a tripod, were measured based on high-resolution aerial images. 
A digitization process was carried out in a CAD software [19] to measure the geometric elements of the roundabouts.

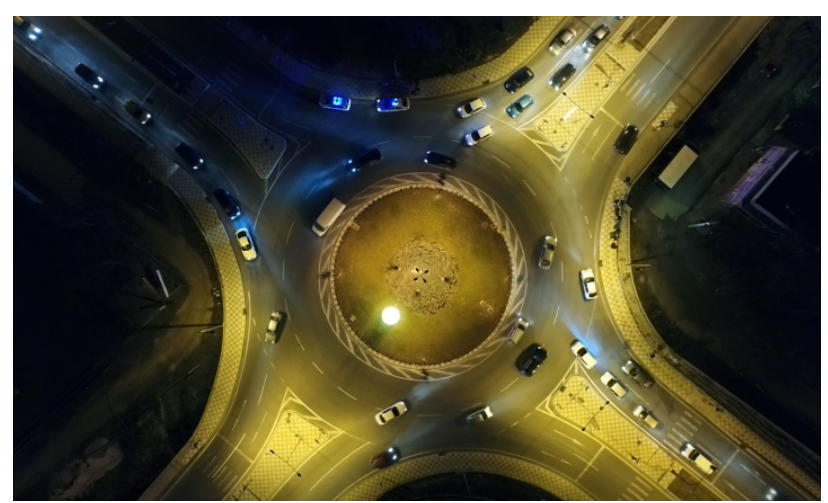

Fig. 2. Field measurements.

\subsubsection{Traffic flow and driving behavior parameters}

After data collection, data required for the neural network modelling (flow rate) were extracted from videos. Oneminute data bins (269 one-minute observations in total) of entry flow and conflicting (circulating) flow were developed for entry lanes, during periods of oversaturation. Follow-up headways and critical headways were extracted from the videos as well for a significant number of the selected roundabouts. Video processing was carried out manually by collecting timestamps at specific events by using the video editing software. Timestamps and events were subsequently inserted in Microsoft Excel for the purpose of calculating the follow-up and critical headway values required. One of the major requirements was the extraction of data when queues were present. As a result, the selected for analysis time periods were minimized. The flow rate of the six events were extracted from the videos using keystrokes at required timestamp as is described in [10].

\section{Methodological Approach}

The current research is aimed at the development of reliable and efficient neural network models for the prediction of actual entry capacity at roundabouts in Greece. The methodology process is described in more detail in the following sections.

\subsection{Characteristics of the Dataset}

The database included data from 24 entry lanes of 11 roundabouts. The statistical software IBM SPSS Statistics [20] was used to proceed in the subsequent analyses. The database consists of 269 cases (one-minute observations of entry flow) and 17 variables. Most of these variables were measured as quantitative and where possible, transformed into qualitative to facilitate additional statistical analyses.

The SPSS database was organized to record all the available variables. As mentioned above, variables are characterized as quantitative (numerical values) and qualitative (ordinal values). Table 2 depicts the available variables as were measured on the field.

Table 2. Variables included in the database.

\begin{tabular}{c|c}
\hline Quantitative variables & Qualitative variables \\
\hline Entry flow & $\begin{array}{c}\text { Intervals of \% entry heavy } \\
\text { vehicles }\end{array}$
\end{tabular}

\section{Circulating flow}

Follow-up headway $\left(\mathrm{t}_{\mathrm{f}}\right)$

Critical headway $\left(\mathrm{t}_{\mathrm{c}}\right)$

Entry width

Entry radius

Entry angle

Circulation width Diameter

Truck apron width Central island

The descriptive statistics of the sample are presented in the following table.

Table 3. Sample descriptive statistics.

\begin{tabular}{c|c|c|c|c}
\hline Variable & Min & Max & Mean & $\begin{array}{c}\text { Std. } \\
\text { Dv. }\end{array}$ \\
\hline Entry flow & 60 & 1,560 & 730.2 & 275.3 \\
Circ. flow & 60 & 1,380 & 520.3 & 303.3 \\
Follow-up headway & 2.2 & 3.4 & 2.8 & 0.30 \\
( $\mathrm{t}_{\mathrm{f}}$ ) & & & & \\
Critical headway $\left(\mathrm{t}_{\mathrm{c}}\right)$ & 3.7 & 5.3 & 4.4 & 0.32 \\
Entry width & 2.8 & 11.7 & 5.6 & 1.99 \\
Entry radius & 4.4 & 53.5 & 16.99 & 11.23 \\
Entry angle & 6.0 & 72.0 & 32.97 & 18.76 \\
Circ. width & 6.0 & 10.0 & 7.47 & 1.45 \\
Diameter & 24.0 & 70.0 & 40.76 & 15.33 \\
Truck apron & 0.0 & 5.0 & 2.26 & 1.61 \\
Central island & 12.0 & 55.0 & 25.81 & 14.19 \\
\hline
\end{tabular}

The values of the IBM SPSS Statistics database were used to identify the correlations among the independent quantitative variables and actual entry lane capacity of roundabouts. Pearson correlation coefficient and the significance value are indicating factors for the evaluation of the correlation analysis' results [21]. The following Table presents the correlations between entry capacity and each of the predictor variables. It is noted that Pearson correlation coefficient takes on values close to 1 for strong relationships and -1 for adverse strong relationships. Moreover, significance values less than 0.05 reveal strong correlation. The two-tailed test of significance was selected for the correlation analysis. It is mentioned that values along with ** are significant at 0.01 level and values along with $*$ are significant at 0.05 level.

Table 4. Results from the bivariate correlation analysis.

\begin{tabular}{c|c|c}
\hline Variables & Code & Pearson correlations \\
\hline Circulating flow & V1 & $-.681^{* *}$ \\
Follow-up headway & V2 & $-.415^{* *}$ \\
$\left(\mathrm{t}_{\mathrm{f}}\right)$ & & $-.169^{* *}$ \\
Critical headway $\left(\mathrm{t}_{\mathrm{c}}\right)$ & $\mathrm{V} 3$ & -.107 \\
\% Entry heavy & V4 & $-.124^{*}$ \\
vehicles & & -.090 \\
\% Circ. heavy vehicles & V5 & $-.139^{*}$ \\
Type of roundabout & V6 & -.031 \\
Code of Roundabout & V7 & $-.204^{* *}$ \\
Lane configuration & V8 & -.031 \\
Lane approach & V9 & -.038 \\
Entry width & V10 & -.083 \\
Entry radius & V11 & -.100 \\
Entry angle & V12 & $.238^{* *}$ \\
Circulation width & V13 & V14
\end{tabular}




\begin{tabular}{c|c|c} 
Truck apron width & V15 & -.098 \\
Central island & V16 & $.279^{* *}$ \\
Location & V17 & -.100
\end{tabular}

According to the correlation analysis results, it is revealed that actual entry lane traffic flow is strongly associated in descending correlating degree with the following variables:

- Circulating traffic flow

- Follow-up headway

- Critical headway

- Lane approach

- Diameter

- Central island

\section{Neural Network Modelling}

Artificial neural networks are mathematical models that belong to the category of empirical capacity models. Their architecture consists of hidden layers with various artificial neural cells that contain activation functions. Several studies use artificial neural networks to predict and estimate various variables based on databases [22-23]. However, a limited number of studies is dealing with neural network modelling of entry lane capacity of roundabouts. Estimated capacities of Turkish roundabouts were calculated on [24], while on [25] artificial neural networks were developed and compared to other empirical models regarding capacities of roundabouts in the U.K.

The database of the explanatory variables and capacity flows was used to develop and train Neural Networks in SPSS. Neural networks produced several models. The dependent variable was "actual entry capacity roundabout lane", while 188 data bins were used for training the neural network and 81 data bins were used for testing the produced neural network.

Various models were developed and evaluated based on different methods, architectures and set of explanatory variables. It was found that a simple feed-forward multilayer perceptron Neural Network with hyperbolic tangent activation functions of hidden and output layers was sufficient to account for most of the model fit.

Table 5 compares the different $\mathrm{NN}$ models as developed for each set of explanatory variables. It is noted that the explanatory variables that reveal strong correlation were considered for the analyses of this study.

Table 5. Results from the bivariate correlation analysis.

\begin{tabular}{c|c|c|c}
\hline $\begin{array}{c}\text { NN } \\
\text { Model }\end{array}$ & Variables included & Mean $\mathbf{R}^{\mathbf{2}}$ & $\begin{array}{c}\text { Mean } \\
\text { RMSE }\end{array}$ \\
\hline 1 & V1 - V4, V9, V16 & 0.71 & 147.7 \\
2 & V1 - V4, V16 & 0.70 & 151.4 \\
3 & all (V1 - V17) & 0.77 & 130.6 \\
\hline
\end{tabular}

It was found that the $\mathrm{NN}$ model including all the variables results in better predictions. The RMSE of the proposed NN model is 130.6 and the $\mathrm{R}^{2}$ is 0.77 . Two hidden layers were considered while the weights and biases were optimized through error back-propagation (Figure 3).

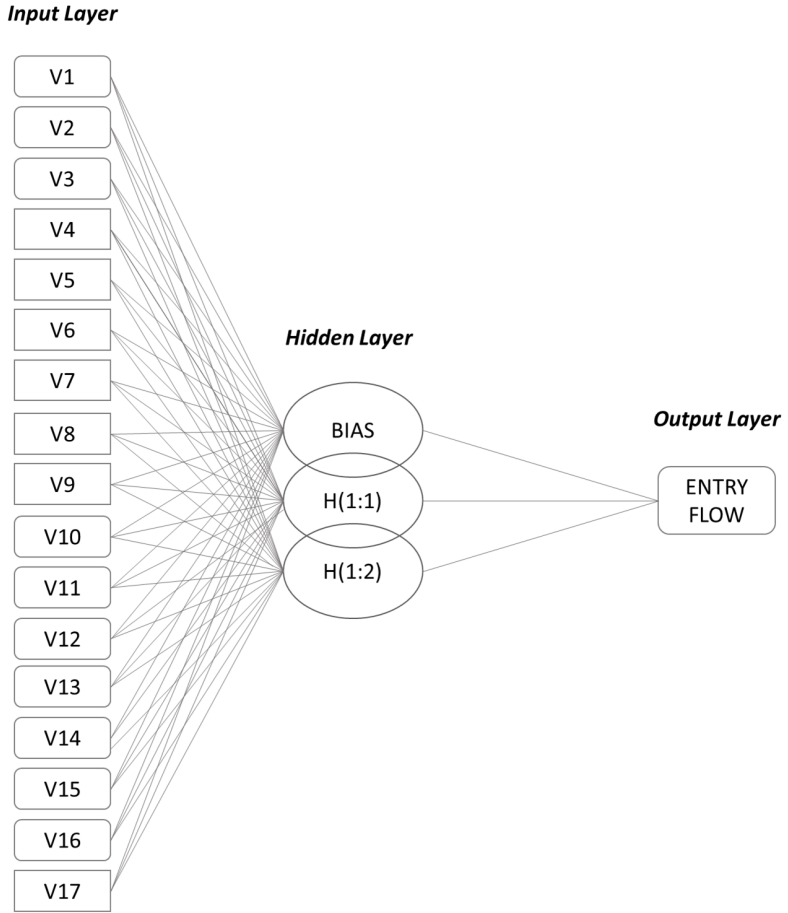

Fig. 3. Neural Network Model with all explanatory variables.

A graphical representation of the actual entry flow and the estimated entry flow according to the developed model is presented in the following Figure.

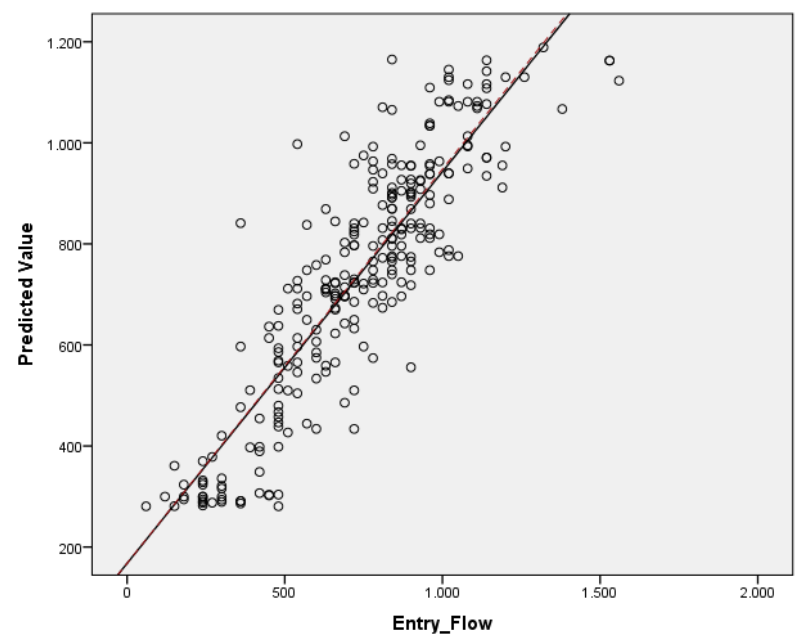

Fig. 4. Actual against predicted capacities.

The following Figure presents the independent variable importance chart. The importance of each variable on how much the network's model-predicted value changes for different values of the independent variables is highlighted.

It appears that the circulating flow has the greatest effect on the capacity of a roundabout lane. Driving behavior and especially the critical headway has a significant impact as well. Finally, the location of the roundabout seems to affect the capacity. This is related to different driving behavior characteristics of drivers among the cities. 


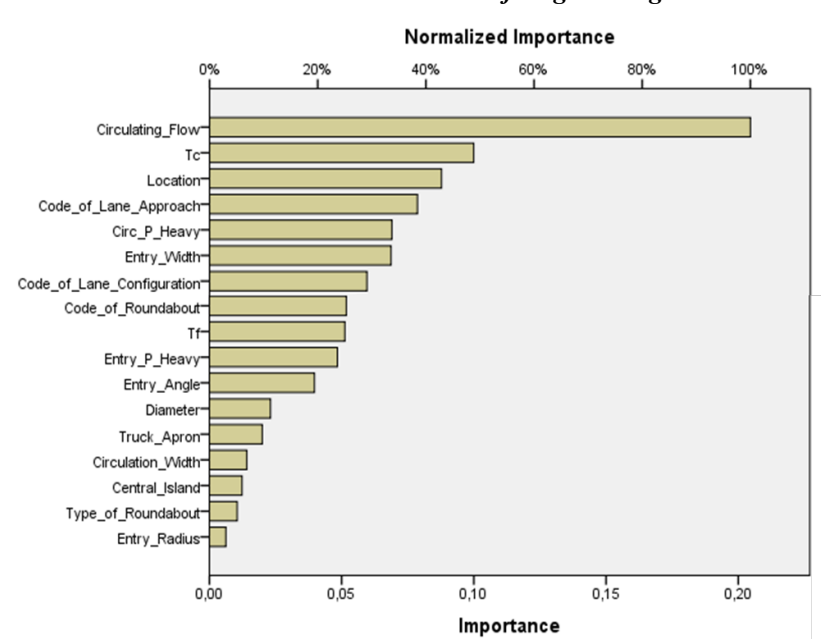

Fig. 5. Independent variable importance.

\section{Conclusions and Future Research}

Accurate roundabout capacity models are essential for optimal roundabout design. As roundabouts have recently being constructed in Greece, drivers gradually get more accustomed to using them which can affect capacity. Upon this, the gradual changes in vehicle characteristics entail that these layouts need to be examined to a wider range of scenarios in terms of road capacity. The proposed methodology addresses the use of machine learning techniques for a proactive operational plan for roundabouts.

An empirical analysis is described in this paper, using traffic flow data under oversaturated periods from 24 entry lanes of roundabouts in Greece.

According to the correlation analysis, it can be concluded that both the geometric characteristics and the driving behavior affect the entry lane capacity of roundabouts. More specifically, the circulating traffic flow and the follow-up headway of vehicles at the entrance of the roundabout are strongly related to the entry traffic flow.

The examination and the development of various neural network models regarding the predictions of entry lane capacity, resulted in valuable conclusions. It was found that a simple feed-forward multilayer perceptron Neural Network with hyperbolic tangent activation functions of hidden and output layers waw sufficient to account for most of the model fit. The RMSE of the proposed NN model is 130.6 and the $\mathrm{R}^{2}$ is 0.77 . Two hidden layers were considered while the weights and biases were optimized through error back-propagation.

According to the results of the developed NN model, it can be concluded that the predictions regarding entry lane capacity of roundabouts are accurate enough. This study is an important step towards developing accurate models of roundabout entry capacity.

Further research is required before the general application of the proposed model in practice. Specifically, an extension and validation of the empirical model is required. More capacity flow measurements and roundabouts through a geographically-wider database will extend the capability of the model.

Moreover, the applicability of the proposed model should be examined in more case studies roundabouts in Greece. The transferability of the model in other countries can be tested as well.

Finally, as the proposed methodology addresses the use of machine learning techniques for a proactive operational plan for roundabouts, the development of NN models per each roundabout category should be examined and compared with the existing roundabout capacity models.

\section{Acknowledgements}

This work was partly supported by the Greek Ministry of Education and Religious Affairs in the scope of the project entitled "Study on the improvement of road safety and capacity of Greek roundabouts, by using emerging technologies and artificial neural networks models" at the Aristotle University of Thessaloniki, project No. 99030. The project was co-funded via the European Social Fund (ESF).

This is an Open Access article distributed under the terms of the Creative Commons Attribution License.

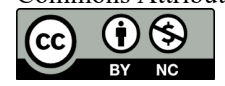

\section{References}

1. Rodegerdts, L., J. Bansen, C. Tiesler, J. Knudsen, E. Myers, M. Johnson, M. Moule, B. Persaud, C. Lyon, S. Hallmark, H. Isebrands, R. B. Crown, B. Guichet \& A. O'Brien. NCHRP Report 672 Roundabouts: An Informational Guide (2nd edition), 2010, Transportation Research Board of the National Academies, Washington, D.C

2. Silva, A. B., L. Vasconelos, \& S. Santos, Moving from Conventional Roundabouts to Turbo-Roundabouts. Procedia Social and Behavioral Sciences, 2014, 111: 137-146.

3. Schoon, C. C., \& J. van Minnen, The Safety of Roundabouts in the Netherlands. Traffic Engineering \& Control, 1994, Vol. 35, No. 3, pp. 142-148.

4. H. L. Khoo and C. Y. Tang, Roundabout system capacity estimation and control strategy with origin-destination pattern, Journal of Transportation Engineering, 2016, vol. 142, no. 5.

5. W. Brilon, Some remarks regarding the estimation of critical gaps, Transportation Research Record, 2016, vol. 2553, no. 1, pp. 1019.

6. Damaskou, E., Kehagia, F., Pitsiava-Latinopoulou, M., and Anagnostopoulos, A. Factors Influencing Quality of Service in Road Safety at Urban Roundabouts, Based on Driver's Perception. Proceedings of the 48th European Transport Conference, 2020.
7. Belz, N. P. Investigating the Role of Turn Indicator Usage by Exiting Vehicles in Gap Acceptance at Single-Lane Roundabouts, 2016, Frontiers of Structural and Civil Engineering, Special Issue: Sustainable Civil Infrastructure.

8. J. Bie, H. K. Lo, and S. C.Wong, Capacity evaluation of multilane traffic roundabout, Journal of Advanced Transportation, 2010, vol. 44, no. 4, pp. 245-255.

9. R. Guo and Y. Zhao, Critical gap of a roundabout based on a logit model, in Proceedings of the Fifth International Conference on Transportation Engineering, 2015, pp. 2597-2603, Dailan, China.

10. Y.H. Yap, H. Gibson and J. Waterson, An International Review of Roundabout Capacity Modelling. Transport Reviews, 2013. 33: p. 593-616.

11. Anagnostopoulos, A., and Kehagia, F. Developing Roundabout Capacity Models in Greece. Proceedings of the 7th Pan-Hellenic Road Safety Conference, 2018, Larissa, Greece.

12. Khan, M.; Ectors, W.T.; Bellemans, D.; Jassens, D.; Wets, G. UAV-Based Traffic Analysis: A Universal Guiding Framework Based on Literature Survey. Transp. Res. Proc. 2017, 22, 541-550.

13. Guido, G., Gallelli, V., Rogano, D., Vitale, A. Evaluating the accuracy of the vehicle tracking data obtained from Unmanned Aerial Vehicles. Int. Journ. of Transp. Sc. and Tech. 2016, 5, 136151 
Apostolos Anagnostopoulos, Fotini Kehagia, Efterpi Damaskou, Anastasios Mouratidis and Georgios Aretoulis/

Journal of Engineering Science and Technology Review 14 (5) (2021) 210 - 215

14. Kanistras, K.; Martins, G., Rutherford, M.J., Valavanis, K.P. Survey of Unmanned Aerial Vehicles (UAVs) for Traffic Monitoring. In Handbook of Unmanned Aerial Vehicles, Valavanis, K., Vachtsevanos G., Eds.; Springer, Dordrecht, pp. 2643-2666.

15. Chen, P., Zeng, W., Yu, G., Wang, Y. Surrogate Safety Analysis of Pedestrian-Vehicle Conflict at Intersections Using Unmanned Aerial Vehicle Videos. Journ. of Adv. Transp., 2017.

16. Outay, F., Mengash, H.A., Adnan, M. Applications of unmanned aerial vehicle (UAV) in road safety, traffic and highway infrastructure management: Recent advances and challenges. Transp. Res. Part A: Policy and Practice, 2020, 141, 116-129.

17. Anagnostopoulos A, Kehagia F. Utilizing UAVs Technology on Microscopic Traffic Naturalistic Data Acquirement. Infrastructures. 2021; 6(6):89.

18. Google Maps. Available online: http:// https://www.google.gr/maps (accessed on 20/07/2021).
19. Autodesk, AutoCAD Software

20. IBM Corporation, IBM SPSS Statistcis (Version 23), Software, IBM.

21. Field, A., Discopering Statistics Using SPSS, 3rd ed., 2009, Sage, London.

22. Aretoulis, G. Neural network models for actual cost prediction in Greek public highway projects. Int. J. Project Organisation and Management. 2019, Vol. 11, No. 1.

23. Titirla, M. and Aretoulis, G. Neural network models for actual duration of Greek highway projects. Journal of Engineering, Design and Technology. 2019, Vol. 17 No. 6, pp. 1323-1339.

24. Ozuysal, M., Caliskanelli, S., Tanyel, S. and Baran, T. Capacity prediction for traffic circles: applicability of ANN. Transport, 2009, 162 (TR4), pp. 195-206.

25. Yap, Y., Gibson, H. and Waterson, B. Models of Roundabout Lane Capacity, Journal of Transportation Engineering, American Society of Civil Engineers, 2015. 